\title{
Migrations africaines et nouveaux enjeux de la frontière israélo-égyptienne
}

African migrations and the new stakes of the Israeli-Egyptian border Migraciones africanas y nuevas problemáticas ligadas a la frontera entre Israel y Egipto

\section{Lisa ANTEBY-YEMINI}

\section{(2) OpenEdition}

\section{Édition électronique}

URL : http://journals.openedition.org/conflits/17307

DOI : $10.4000 /$ conflits. 17307

ISSN : $1777-5345$

Éditeur :

CCLS - Centre d'études sur les conflits lilberté et sécurité, L'Harmattan

\section{Édition imprimée}

Date de publication : 20 décembre 2008

Pagination : 77-99

ISBN : 1157-966X

ISSN : 1157-996X

Référence électronique

Lisa ANTEBY-YEMINI, « Migrations africaines et nouveaux enjeux de la frontière israélo-égyptienne », Cultures \& Conflits [En ligne], 72 | hiver 2008, mis en ligne le 19 mai 2009, consulté le 30 mars 2021.

URL : http://journals.openedition.org/conflits/17307 ; DOI : https://doi.org/10.4000/conflits. 17307 


\section{Migrations africaines et nouveaux enjeux de la frontière israélo-égyptienne}

\section{Lisa ANTEBY-YEMINI}

Lisa Anteby-Yemini est anthropologue, chargée de recherche au CNRS et membre de l'Institut d'ethnologie méditerranéenne, européenne et comparative (IDEMEC) à Aix-en-Provence. A paraître : "Les "réfugiés soudanais" en Israël: discours, représentations, mobilisations ", Maghreb-Machrek, 2009.

Traël, souvent présenté comme un pays aux frontières hermétiques, en parIiculier médiatisées par la construction du Mur de séparation avec les Territoires palestiniens, possède également une frontière longue de $220 \mathrm{~km}$ avec l'Egypte, qui semble, quant à elle, fort poreuse à bien des égards. Il existe encore peu de travaux sur cette frontière, bien que cette dernière suscite un intérêt croissant depuis quelques années, avec le passage clandestin de migrants économiques et de demandeurs d'asile, mais aussi en raison de l'intensification de trafics d'êtres humains.

Cette zone frontalière entre l'Egypte et Israël, qui traverse le désert du Sinaï, est peu peuplée. Aux points-frontières de Taba (à l'extrême Sud) ou de Nizzana (au Nord), peu de passages réguliers sont enregistrés. Quelques Egyptiens, coptes pour la plupart, se rendent en pèlerinage aux lieux saints à Jérusalem, tandis qu'à certaines périodes, quelques milliers d'Israéliens traversent la frontière à Taba pour passer des vacances dans le Sinaï, au bord de la Mer Rouge. Il n'y a donc pas de véritable « culture de la frontière » ni de ville-frontière (à l'exception de Rafah, située entre l'Egypte et la bande de Gaza). Par conséquent, il n'existe pas de circulations intensives ni d'échanges commerciaux comme on en trouve entre Israël et les Territoires palestiniens. En revanche, une ancienne tradition de commerce informel existe, en particulier parmi les Bédouins qui, désormais, se « reconvertissent » en passeurs d'êtres humains. Aussi, le passage se fait-il à chaque fois dans un seul sens : le tourisme d'Israël vers l'Egypte, et la circulation clandestine de biens et de 
personnes d'Egypte vers Israël. On peut donc parler ici, pour emprunter l'expression de Donnan et Wilson, de «zone frontière aliénée 1 " («alienated borderland») où l'échange routinier transfrontalier est absent en raison des tensions existant entre les deux Etats et où le contact quotidien dans ces zones frontalières est minimal, sinon inexistant.

C'est dans ce contexte que commence, surtout au début de l'année 2000, le passage de migrants vers Israël, en majorité originaires d'Afrique subsaharienne. Nous allons voir comment, en l'espace de quelques années, cette frontière peu traversée, en dehors de contrebandiers bédouins, est devenue le point principal d'entrée illégale vers le territoire israélien. Jusqu'à la fin des années 1990, par le biais d'agences de recrutement ou de visas touristiques, des migrants de travail étaient arrivés quasi exclusivement par l'aéroport BenGurion de Tel-Aviv, en provenance de pays aussi divers que la Chine, la Turquie, la Roumanie, la Thaillande, le Ghana ou la Colombie 2. Désormais, ceux qui passent par la frontière israélo-égyptienne n'ont pas d'autre choix que de venir par voie terrestre clandestine, n'ayant pas accès à des visas de touristes ou de travail. Par quel biais Israël est-il alors venu s'inscrire dans les destinations migratoires de populations qui n'avaient jamais envisagé de rejoindre ce pays ? En un mot, comment cette frontière, perçue en Israël comme une frontière sécuritaire, est-elle devenue aujourd'hui également une frontière de migration irrégulière entre l'Afrique et Israël ?

Divers facteurs peuvent expliquer ces développements, comme la recherche d'une nouvelle terre d'asile pour les réfugiés soudanais, qui ne se sentent plus en sécurité en Egypte depuis la répression de la police égyptienne, sous les bureaux du Haut Commissariat pour les réfugiés de l'ONU (HCR), au Caire en 2005. De même, l'ouverture en 1999 d'une représentation du HCR à Jérusalem et, en 2007, d'un bureau à Tel-Aviv a également décidé certains demandeurs d'asile à migrer vers Israël. Mais ce sont surtout des mesures prises par l'Etat israélien, telles que l'octroi de permis de résidence à 600 Soudanais du Darfour et de protections temporaires (parfois couplées au permis de travail) aux Erythréens, Ivoiriens et Congolais (de RDC), qui encouragent ces circulations. Enfin, rappelons que ces itinéraires migratoires suivent d'anciennes routes de contrebande et qu'ils ne font donc que réactualiser les commerces informels, déjà anciens, en une économie parallèle de droit de passage.

Il s'agit donc de comprendre par quels dispositifs Israël, avec un régime frontalier si fermé, surtout au vu des dispositifs existants à l'encontre des Palestiniens, met progressivement en place, sur cette «frontière floue » (bien

1. Donnan H., Wilson T., Borders: Frontiers of Identity, Nation and State, Oxford, Berg, 2001, p. 51.

2. Voir Berthomière W., « Nouvelle Intifada, mondialisation et immigration de travailleurs étrangers : une nouvelle réalité sociale pour Israël », Maghreb-Machrek, n¹77, 2003, pp. 79-99. 
que marquée géographiquement), un système de contrôle, de surveillance et de rétention pour ceux qui la franchissent. Dans un pays où les frontières sont un enjeu politique et stratégique majeur, cette frontière constitue donc un nouvel enjeu, d'une part pour ceux qui tentent de la traverser et qui la perçoivent comme une démarcation entre pays «pauvre » et pays « riche », pays qui «opprime les migrants» et pays «des droits de l'homme » et, d'autre part, pour l'Etat d'Israël, qui se voit confronté à un flot sans précédent de demandeurs d'asile africains, et qui doit faire face pour la première fois à un défi de taille en matière de gestion de ces flux et de politique d'asile, quasi inexistante à ce jour ${ }^{3}$.

\section{L'économie de la frontière}

Avant de poursuivre, il nous faut revenir sur les distinctions opérées entre migrants économiques, demandeurs d'asile et réfugiés, termes utilisés pour décrire ceux qui entrent en Israël par l'Egypte. En Europe, on qualifie de plus en plus les réfugiés de «faux » demandeurs d'asile, créant une hiérarchie entre victimes de persécutions "politiques » et celles dites «économiques ", d'où le développement du concept de «demandeur illégal d'asile 4 ». En Israël, l'expression « réfugiés africains » est la plus courante pour désigner ces migrants, sans pour autant faire référence à leur statut juridique réel ${ }^{5}$, alors que le discours officiel fait souvent référence à la législation qui définit tous ceux qui traversent clandestinement la frontière israélo-égyptienne comme des «infiltrateurs » (mistanenim). Nous allons opter dans cet article pour la dénomination «migrants forcés » qui nous paraît plus neutre, compte tenu des divers motifs de la migration.

\section{Qui sont les «infiltrateurs»?}

Les chiffres sont en augmentation rapide depuis l'été 2007, moment où un record a été atteint avec un chiffre, pour le seul mois de juillet, de 900 personnes entrées clandestinement sur le territoire israélien par la frontière avec l'Egypte, soit un total de 5000 personnes pour 2007, les Soudanais étant cette année-là le groupe le plus représenté ; pour 2008, on compte 7500 demandeurs d'asile passés par la frontière israélo-égyptienne ${ }^{6}$. Aujourd'hui, on

3. Cette recherche, menée depuis 2007, est issue d'entretiens avec ces migrants forcés, qui ont eu lieu dans des abris d'urgence, des cybercafés, des églises et des bars à Tel-Aviv, avec un accent particulier mis sur la traversée de la frontière et le choix d'Israël comme destination. De plus, une activité de bénévolat auprès d'une ONG israélienne d'assistance juridique (Hotline for Migrant Workers) nous a permis de comprendre les rouages institutionnels à l'œuvre dans le traitement de migrants forcés et de rendre visite à ceux détenus en prison.

4. Morice A., Rodier C., "Classer-trier migrants et réfugiés : des distinctions qui font mal ", Hommes et libertés. Revue de la Ligue des droits de l'Homme, n²9, 2005 pp. 58-61.

5 . Voir Anteby-Yemini L., "Les "réfugiés soudanais" en Israël : discours, représentations, mobilisations », Maghreb-Machrek, à paraître en janvier 2009.

6. Shahar I., "Number of Illegal Migrant Workers Hits 300,000”, Ha'aretz, 14 janvier 2008. 
dénombre près de 14000 demandeurs d'asile en Israël, en majorité d'origine africaine ${ }^{7}$; plus de 6500 sont originaires d'Erythrée, 5000 du Soudan (dont une majorité de chrétiens du Sud-Soudan, environ 1500 musulmans du Darfour et une centaine provenant de la région des monts Nuba), près de 1000 de Côte d'Ivoire, 500 de la République démocratique du Congo et, en nombre plus restreint, d'Ethiopie, de Somalie, de Guinée-Conakry, du Togo, et du Tchad notamment, dont les premiers sont arrivés dès les années 1990. A une majorité de candidats à l'asile politique, tels les Erythréens qui désertent $\mathrm{du}$ service militaire obligatoire et sans limite de temps ou les Soudanais qui fuient des persécutions religieuses ou ethniques, s'ajoutent également des migrants de travail, en provenance de Colombie ou de Chine par exemple, ainsi que des victimes de trafic d'êtres humains et de réseaux de prostitution (en général, des femmes des pays d'Europe de l'Est), qui empruntent la même route, mais dont nous ne traiterons pas ici.

Parmi les migrants forcés subsahariens, certains ont voyagé durant de longues années pour atteindre l'Egypte, puis Israël, d'autres ont vécu plusieurs années au Soudan (comme certains Erythréens ou Ethiopiens) de même qu'en Egypte, et d'autres encore ont quitté leur pays d'origine pour arriver quelques semaines plus tard en Israël. Ainsi, Bernard ${ }^{8}$ a fui de la RDC vers le Mali, puis est arrivé en Algérie et enfin au Maroc ; là il a été arrêté et refoulé en Algérie où il a été emprisonné puis expulsé au Mali. Il a ensuite acheté un faux passeport ivoirien pour pouvoir se rendre directement au Caire sans visa et a finalement atteint la frontière israélienne. Louis, un Ivoirien échappé d'un camp de réfugiés en République de Guinée, est ensuite allé au Burkina-Faso, puis a travaillé un an en Libye pour continuer sa route vers l'Egypte, où il est resté quelques semaines, avant d'arriver en Israël. Dans leur majorité, il s'agit de jeunes hommes, souvent instruits ; mais on constate aujourd'hui que de plus en plus de femmes tentent leur chance (souvent accompagnées d'enfants en bas âge et ayant parfois laissé leur conjoint en Egypte ou ailleurs), ainsi que des mineurs non accompagnés.

\section{Israël : un nowveau paradis?}

Selon les estimations, il y a entre 500000 et 3 millions de réfugiés et demandeurs d'asile en Egypte, principalement des Palestiniens, des Soudanais, des Somaliens, des Ethiopiens et des Erythréens, mais aussi des personnes venant du Libéria, de Sierra Leone, du Yémen, du Burundi et d'Afghanistan 9. Leurs conditions de vie sont souvent très précaires, avec des restrictions d'accès au

7. Rapport du Forum israélien pour les Droits des réfugiés novembre 2008 : www.hotline.org.il/english/refugees_rights_forum.htm

8 . Les prénoms de nos interlocuteurs ont été modifiés.

9. Voir Zohry A., "The Place of Egypt in the Regional Migration System as a Receiving Country”, Revue européenne des migrations internationales, $\mathrm{n}^{\circ} 3$, vol.19, 2003, pp. 129-149. 
marché du travail, des obstacles à la scolarisation des enfants, une pauvreté rampante et la menace permanente de la détention et de l'expulsion ${ }^{10}$. En plus du racisme, des abus et de la discrimination dont font l'objet les Subsahariens en Egypte 11, c'est la brutalité de la police égyptienne lors d'une manifestation près des bureaux du HCR au Caire, en décembre 2005 (où, selon les sources, entre 27 d'après les autorités et 200 demandeurs d'asile et réfugiés soudanais ont été tués ${ }^{12}$ ), qui a précipité la recherche de nouvelles routes migratoires vers des destinations porteuses d'un avenir meilleur. Outre les programmes de réinstallation vers l'Australie, le Canada, la Finlande ou les Etats-Unis, la voie vers la Libye, avec l'espoir d'atteindre l'Europe, s'est de plus en plus développée pour ceux qui n'avaient pas de perspective d'être réinstallés. C'est aussi pourquoi, depuis peu, Israël s'impose comme une nouvelle destination dans ces itinéraires d'exil. Or, parce que la plupart des Subsahariens n'auraient jamais pu obtenir de visa pour entrer légalement en Israël, ils optent pour l'entrée clandestine par la frontière israélo-égyptienne. Cette frontière représente, pour eux, la garantie d'une protection, le rêve de la prospérité et l'assurance de vivre dans un pays "démocratique " qui prend peu à peu l'image d'une terre «d'asile », voire d'une terre «promise »... Mais c'est aussi sur les traces d'un trafic ancien qu'ils réactualisent ces itinéraires de passages informels.

\section{D'anciennes routes de commerce illicite}

Le Sinaï est depuis longue date le lieu de trafics de marchandises, de drogue et d'alcool entre l'Egypte, la bande de Gaza et Israël, mais également entre la Jordanie, la Syrie et même l'Irak. Ce sont tout d'abord les Bédouins de la région qui ont développé, après 1948, des réseaux de contrebande, comme celui du bétail dans les années 1960, dans ces zones peu surveillées, tout en ayant le consentement officieux des autorités israéliennes ${ }^{13}$. Une véritable économie informelle s'est ainsi mise en place avec des revenus non négligeables, pour les populations bédouines en particulier. Aujourd'hui, ces mêmes contrebandiers font passer des travailleurs palestiniens des Territoires occupés vers Israël ou sont impliqués dans le trafic de drogue entre l'Egypte, Israël, la bande de Gaza et les Territoires occupés ${ }^{14}$. Il était donc naturel que les Bédouins du Sinaï soient aussi devenus des passeurs, prenant part au trafic d'êtres humains qui a commencé dans les années 1990 avec le passage clandestin d'Egypte vers Israël de femmes des pays de l'Est pour le compte de réseaux internationaux de prostitution. Les autorités israéliennes auraient pu contrôler ces flux, mais elles ont

10. Ibid., pp. 138-139.

11. Sur le racisme envers les Soudanais en Egypte, voir Le Houérou F., «A la rencontre des mondes : l'épopée des réfugiés du Darfour », Maghreb-Machrek, n¹85, 2005, pp. 103-126.

12. Voir Harrell-Bond B. (interrogée par F. Le Houérou), «Le drame de la place Mustapha Mahmoud au Caire », disponible sur : http://terra.rezo.net/article553.html

13. Parizot C., " Gaza, Beer Sheva, Dhahariyya : les relations entre les bédouins et l'Etat d'Israël », Bulletin du Centre de recherche français de Jérusalem, ${ }^{\circ} 9,2001$, pp. 37-50.

14 . Ibid., p. 49. 
plutôt adopté une position de tolérance et même de collaboration, dans la mesure où ces passages ne représentaient pas une menace pour la sécurité $\mathrm{du}$ pays et n'impliquaient pas d'armes ou de complicités avec des visées terroristes ${ }^{15}$. Mais, bien avant le début des années 2000 , un autre trafic d'êtres humains s'est développé : le passage de migrants forcés. Toutefois, il est ici parfois difficile de faire la distinction entre trafic d'êtres humains et simple prix de passage clandestin (autrement dit, la différence entre smuggling et trafficking) ${ }^{16}$ puisque, si la majorité des migrants forcés (du Soudan, d'Erythrée ou de Côte d'Ivoire) paient directement à des passeurs en Egypte une somme allant de 500 à 3500 dollars pour être menés jusqu'à la frontière, d'autres sont parfois contraints de travailler dans des conditions proches de celle de l'esclavage moderne en Israël pour rembourser le prix du passage.

C'est ainsi que, lorsque de plus en plus de migrants forcés en Egypte décident de tenter leur chance en Israël, les Bédouins profitent de cette nouvelle opportunité économique en développant des réseaux de passeurs jusqu'à la frontière israélienne. En effet, ces circulations récentes se calquent sur les anciennes routes stratégiques de contrebande et les passeurs mettent à profit leur «savoir circuler » dans ce terrain désertique, de même que l'on retrouve aujourd'hui au Sahara les routes migratoires actuelles plaquées sur les anciens itinéraires d'échange et l'instrumentalisation par les migrants du savoir des populations nomades touaregs ${ }^{17}$. Comme sur d'autres frontières, entre Israël et les Territoires palestiniens par exemple, les Bédouins détiennent le monopole sur le trafic de migration informelle et imposent des prix élevés aux candidats au passage ${ }^{18}$. Ainsi, aujourd'hui, dans la continuité historique de ces pratiques de transgression liées au trafic bédouin et en sus de la contrebande, la frontière elle-même et son passage deviennent une ressource économique lucrative pour les Bédouins d'Egypte. Cette économie frontalière est d'ailleurs de plus en plus organisée en amont pour appâter les candidats à la traversée.

\section{Des contrebandiers reconvertis en passeurs}

C’est généralement au Caire que s'organisent les départs. Il est certain que ce sont à la fois les rumeurs que font courir les Bédouins sur la situation « dorée » en Israël et leurs méthodes de « recrutement » qui encouragent ce

15. Suite à une loi votée en 2004 par le Parlement israélien qui sanctionne le trafic d'êtres humains, on observe un durcissement du contrôle de ces passages.

16 . «le trafic de personnes signifie le recrutement, le transport ou la réception de personnes par la menace on l'usage de la force on d'antres formes de coercition, de frande, d'abus de ponvoir on d'une position de vulnérabilité ou le fait de donner ou recevoir un paiement pour qu'une personne consente à être contrôlée par une autre pour l'exploitation. " (ONU, Protocole visant à prévenir, réprimer et punir la traite des personnes, en particulier des femmes et des enfants, 2000 , résolution $55 / 25$ de l'Assemblée générale).

17. Bensaad A., «Les migrations transsahariennes, une mondialisation par la marge », MaghrebMachrek, n'185, 2005, pp. 13-36.

18 . Parizot C., "Tightening Closure, Securing Disorder”, Refugee Watch, n⿳31, 2008, pp. 54-74. 
flux de migrants forcés vers Israël, indépendamment des facteurs évoqués plus haut concernant la recherche de routes pour quitter l'Egypte.

Ces passeurs professionnels rencontrent leurs « clients» soit dans des cafés cairotes, par relations dans les milieux fréquentés par les migrants, soit ils contactent directement les candidats au passage. A ce titre, Stéphane, un jeune Ivoirien parti pour le Maroc, n'y a pas trouvé de travail et décide, après quelques semaines, de continuer sa route vers l'Egypte. Après plusieurs mois au Caire, il se trouve toujours dans une situation économique difficile lorsqu'il entend parler d'Israël par d'autres Ivoiriens et décide d'y tenter sa chance. Il « marchande » son passage avec les passeurs, en plaidant qu'il ne lui reste presque rien et ne paie que 500 dollars; jamais il n'avait pensé migrer en Israël. Ahmed, quant à lui, n'avait jamais entendu parler d'Israël à Conakry jusqu'à ce qu'il arrive dans la capitale égyptienne. De même, ce n'est qu'au Caire que beaucoup d'Erythréens rencontrent des compatriotes qui les enjoignent à les suivre en Israël, alors que cette destination n'avait pas été particulièrement envisagée auparavant. C'est aussi le cas de Soudanais comme Marc, originaire du Sud-Soudan, qui a vécu pendant sept ans au Caire, survivant grâce à de petits boulots, jusqu'à ce qu'un Bédouin l'appelle pour lui proposer de le faire passer en Israël. Persuadé qu'il s'agit d'un agent secret soudanais, il ne répond pas aux appels suivants, mais l'insistance du passeur le décide à le rencontrer, comprenant qu'un ami parti en Israël lui avait donné son numéro. Les contacts avec les passeurs fonctionnent donc suivant une logique de réseau. En outre, des informations circulent parmi les communautés africaines du Caire concernant les passeurs, leurs compétences et leurs prix.

En réalité, plusieurs migrants forcés soulignent que le prix du passage, fixé au Caire, dépend du point de la frontière où les passeurs les mènent : en fonction de la somme que l'on paie, il existe des endroits plus faciles à traverser et donc plus chers que d'autres. Il existe également des réseaux plus vastes dans lesquels les passeurs bédouins ne sont qu'un maillon d'une filière qui commence au pays d'origine et qui se termine généralement à Tel-Aviv. Par exemple, certains de nos interlocuteurs nous ont parlé d'entremetteurs chinois en Israël qui faisaient venir des femmes de leur pays à des fins d'exploitation sexuelle. D'autres nous ont parlé d'un Guinéen à Conakry qui leur promettait un emploi en Israël contre 2000 dollars, à rembourser une fois dans le pays ; les voyages par avion via l'Egypte sont arrangés à l'avance, et des Bédouins attendent le groupe à l'aéroport du Caire afin de les conduire dans le Sinaï. Une fois la frontière franchie, du côté israélien, attendent d'autres Bédouins qui les conduisent jusqu'à Tel-Aviv où ils devaient travailler plusieurs mois pour payer à un entremetteur d'origine guinéenne la somme exigée. Néanmoins, ces « trajets organisés » ne concernent qu'une minorité de passages.

Le Caire est donc une plaque tournante pour poursuivre la migration, que ce soit vers la Libye ou vers Israël. Les passeurs et trafiquants bédouins qui 
vivent de la frontière et de son passage appartiennent à des réseaux connus pour leurs activités traditionnelles de commerce illicite, comme ailleurs où les « guides » proviennent de populations impliquées dans des activités clandestines frontalières. Ces passages engendrent ainsi de nouvelles formes de criminalité qui octroient aux Bédouins un rôle de pouvoir dans ces circulations vers Israël. C'est donc par la dynamique impulsée par les nouvelles populations qui traversent cette ligne de démarcation (demandeurs d'asile, migrants économiques, victimes de trafic d'êtres humains) et par les anciennes, qui en régulent le passage, que cette frontière se transforme peu à peu en frontière migratoire.

\section{La violence de la frontière}

La frontière israélo-égyptienne, qui passe dans une zone désertique du Sinaï, n'est pas seulement un axe de contrebande et d'immigration clandestine, elle est aussi une entrée pour des terroristes potentiels sortant de la bande de Gaza pour pénétrer en Israël. La fonction première de cette frontière pour l'Etat d'Israël est donc une fonction sécuritaire, priorité des patrouilles de l'armée israélienne postées le long de cette ligne de démarcation. Or, depuis l'afflux de migrants forcés qui la traversent, l'Etat israélien fait pression sur l'Egypte afin d'obtenir qu'elle contrôle ces circulations. Le Premier ministre israélien, Ehud Olmert, tenait un discours de plus en plus ferme concernant une «invasion d'infiltrateurs de travail » (mistanenei avoda, en hébreu), en qualifiant dans un discours de mars 2008 de «tsunami » l'augmentation récente de «réfugiés africains » entrés en Israël ${ }^{19}$. De ce fait, les patrouilles de sécurité égyptiennes ont été renforcées dans les zones frontalières, bien que la longueur de celle-ci et les astuces des passeurs empêchent une surveillance parfaite. Néanmoins, dans ce contexte, la traversée de la frontière donne lieu à diverses formes de violence (harcèlement et abus sexuels de la part des Bédouins, faim et soif, obstacles physiques, tirs des soldats égyptiens, blessures causées par les barbelés, séparation ou perte d'un proche) sur lesquelles nous nous arrêterons.

\section{La traversée et ses périls}

Le parcours dans le désert à partir du Caire réserve aux migrants son lot de dangers et de violence, aussi bien physique que symbolique. Tous racontent les jours, voire les semaines, passés dans le désert du Sinaï comme une douloureuse étape du voyage, dont on ne sort pas indemne. C'est le plus souvent en voiture ou en camionnette, parfois à dos d'âne, que partent les migrants du Caire, en compagnie des passeurs, pour le désert. Il y a ensuite parfois une longue marche à pied avant d'atteindre une bâtisse dans le désert où sont confinés les migrants pour un temps indéterminé (de quelques jours à

19. Frenkel S., “African Refugees to be Deported at Point of Arrival”, Jerusalem Post, 24 mars 2008. 
un mois). Wolde, qui s'était enfui deux semaines plus tôt d'Erythrée, se souvient qu'on lui avait raconté que tout le trajet se faisait en voiture, alors qu'il a dû marcher plusieurs heures à pied; d'autres évoquent une marche de plusieurs jours dans le désert. Certains se représentent un espace où il est aisé de traverser les frontières, tel Wali, un jeune Togolais, sûr qu'on allait en voiture sans arrêt du Caire à Tel-Aviv, comme du Togo au Bénin. Les Bédouins n'offrent pas toujours de vivres ; Marie, une Tchadienne, affirme ainsi ne pas avoir mangé durant trois jours. Mais, hormis la faim, la soif, la chaleur et le confinement dans l'attente du passage, c'est le harcèlement des Bédouins qui marque les migrants. Beaucoup racontent qu'ils devaient remettre aux passeurs tous leurs documents, passeport, valises et argent sous la menace du fusil ; des femmes parlent encore difficilement de viols et autres abus sexuels commis par ces mêmes passeurs.

Lorsque ces " guides » décident que le temps est venu de tenter le passage, ce sont en général des groupes d'une dizaine de migrants, souvent de nationalités différentes, qui partent au milieu de la nuit, pour avoir davantage de chances de ne pas être repérés. Beaucoup rapportent qu'il leur a fallu plusieurs tentatives pour franchir la frontière et, lorsque les passeurs décident que la route n'est pas bonne ou que la surveillance est trop aigüe, le groupe est reconduit à la bâtisse de départ. Ahmed, de Guinée-Conakry, se souvient que le passeur les a emmenés trois fois vers la frontière mais, à chaque fois, ils ont dû rebrousser chemin. Les points exacts de passage sont multiples, mais se situent tous au Sud de la ville-frontière de Rafah.

Dès que les lumières du côté israélien sont visibles, les Bédouins abandonnent leurs « clients » à leur sort, en leur indiquant la direction à prendre après avoir franchi les grillages de barbelés. Ismail, originaire du Darfour, raconte : "Le passeur m'a dit: tu traverses un grillage, tu marches dans les montagnes, tu traverses un autre grillage et c'est Israël». En effet, la frontière en soi témoigne de peu d'investissements, de matériel de détection, ou d'effectifs de surveillance, et constitue une installation précaire en comparaison avec d'autres frontières dans le monde, telles celles de Ceuta et Melilla ou de certaines parties entre les Etats-Unis et le Mexique, dotées de murs électroniques, de radars ou de caméras infrarouges. Cependant, la porosité de cette frontière aurait été bien plus importante sans la présence des soldats égyptiens.

\section{Brutalité de la « sortie d'Egypte»}

Avec l'accroissement du nombre de migrants forcés qui se dirigent vers Israël depuis l'été 2007 , les autorités égyptiennes ne cessent de répéter qu'ils mènent des efforts "pour lutter contre l'infiltration à travers les frontières internationales avec Israël ». Ces efforts se manifestent effectivement par un contrôle et une surveillance accrus de la zone frontalière mais se traduisent également par une augmentation du nombre de victimes. Car, pour lutter contre 
«l'infiltration en Israël », les soldats sont contraints de lutter contre la sortie du territoire égyptien et donc de contrôler la liberté de circulation des migrants, quitte à utiliser la force. C'est ainsi que la répression ne cesse de s'accroître et nombreux sont les migrants à être arrêtés avant de franchir la frontière, risquant les interrogations, la prison, la torture ou même le rapatriement dans le pays d'origine (qui signifie souvent des peines allant jusqu'à l'exécution). Ainsi, durant l'été 2007, des raids des forces de sécurité égyptiennes dans la région frontalière avec Israël ont mené à l'arrestation de plus de 230 personnes, soudanaises pour la plupart. Depuis, plus de 1300 migrants ont été jugés par des tribunaux militaires égyptiens et condamnés à des peines de prison pour avoir «tenté de passer illégalement la frontière orientale de l'Egypte 20 ». Ceux que les Egyptiens n'arrivent pas à arrêter sont souvent blessés par balle ou, pire, abattus. Ce régime de terreur et cette brutalité excessive à la frontière font l'objet de rapports réguliers par l'ONG Amnesty International qui a appelé récemment le gouvernement égyptien «à protéger les droits fondamentaux des personnes interceptées à la frontière avec Israël suite à la mort de demandeurs d'asile soudanais, érythréens, ivoiriens et guinéens 21 ». Effectivement, près d'une trentaine de femmes et d'hommes sont décédés depuis 2007, tandis que les forces de sécurité égyptiennes ouvraient le feu sur ceux qui tentaient de franchir la frontière et que de nombreux autres, dont des enfants, ont été blessés tandis qu'ils essayaient de sauter au-dessus des barbelés, ignorant les injonctions à s'arrêter.

Samuel, un Somalien habitant Tel-Aviv depuis trois ans, se souvient qu'un de ses amis souffrant n'avait pu courir aussi vite que lui pour sauter par-dessus le grillage : les soldats égyptiens lui ont tiré dessus. Il pense qu'il est mort car il n'a plus jamais eu de ses nouvelles... D'autres racontent que des amis ou des compagnons de voyage ont été arrêtés, blessés ou tués, entraînant des traumatismes évidents pour les survivants. Des drames se produisent également lorsque des familles sont séparées, les uns arrêtés ou blessés du côté égyptien, les autres parvenant jusqu'au côté israélien, ou encore des tragédies humaines comme celle de ce Soudanais rencontré dans un abri d'urgence à Tel-Aviv qui escaladait le grillage de barbelés avec son nourrisson âgé de six mois lorsque les soldats égyptiens ont arrêté sa femme; l'homme, en présence des soldats israéliens arrivés à la frontière, a supplié les Egyptiens de laisser passer sa femme mais ils ont refusé, donnant le choix au père de revenir en Egypte ou de redonner l'enfant à sa mère. Trop inquiet du sort du nourrisson dans une prison égyptienne, l'homme a décidé de rester du côté israélien avec le bébé et sa femme est aujourd'hui détenue près du Caire, menacée de rapatriement... Un autre déchirement familial, qui a pris un tournant plus réjouissant, a fait la

20. Amnesty International, document «Action urgente», daté du 3 septembre 2008. http://www.amnesty.org/fr/library/info/MDE15/038/2008/fr

21. www.amnesty.org/fr/library/asset/MDE12/027/2007/fr/R1hAiBMreZcJ 
« une » de la presse israélienne : une fillette soudanaise restée du côté égyptien alors que ses parents avaient réussi à traverser en Israël avait été retrouvée après plusieurs jours et exceptionnellement remise par les Egyptiens à la frontière afin qu'elle puisse rejoindre ses parents. Ces voyages risqués font d'ailleurs l'objet d'une médiatisation croissante, comme le témoigne la couverture récente du Monde 2 avec la photo d'une famille soudanaise arrêtée en pleine nuit à la frontière par des soldats israéliens et le titre : « La fuite en Israël des réfugiés d'Afrique. Venues du Darfour et d'Erythrée, des milliers de familles tentent au péril de leur vie d'entrer en Israël. Photoreportage sur un drame méconnu $22 »$.

La frontière israélo-égyptienne sépare donc clairement deux côtés - l'un perçu comme hostile, et l'autre comme protecteur - et délimite ainsi deux territoires souverains aux expressions de pouvoir territorial forts différentes. A ce titre, si l'usage disproportionné de la force par l'Egypte peut être analysé en continuité avec la brutalité de la répression policière de 2005 au Caire, la délocalisation de la frontière israélienne par le contrôle égyptien à l'extérieur ou en deçà du territoire israélien doit aussi être prise en compte. Car «les instruments de la flexibilisation des frontières d'un Etat peuvent également être, en somme, les gouvernements d'autres Etats, avec leurs activités de police dont les buts et les modalités d'action sont dictés et contrôlés par les pays de destination 23 ». Ainsi, Israël imposerait ses propres politiques sécuritaires et antimigratoires à son voisin égyptien qui les interprète à sa façon.

\section{L’hospitalité en "Terre promise »}

C'est ainsi que la plupart des migrants forcés arrivent du côté israélien exténués par la course pour échapper aux autorités égyptiennes, et parfois par une longue marche, mais surtout traumatisés par le fait d'avoir perdu ou laissé des proches à la frontière, et enfin, bien souvent blessés eux-mêmes par les tirs égyptiens ou le franchissement des barbelés. En général, les premiers Israéliens qu'ils rencontrent ne sont ni des douaniers ni des policiers, mais les gardes-frontières ou les soldats de l'armée israélienne qui patrouillent en permanence autour de ces espaces frontaliers. Leur mission principale est de surveiller la frontière pour empêcher des tentatives d'infiltration par le Sinaï de groupes armés palestiniens en provenance de la bande de Gaza et ce, surtout durant l'été 2007, lorsque la barrière séparant Gaza de l'Egypte avait été ouverte par des foules palestiniennes pressées de se ravitailler du côté égyptien en raison du blocus israélien imposé à toute la bande de Gaza. Toutefois, pour l'armée israélienne, ces mobilités migratoires relèvent d'un problème civil et

22. Supplément au Monde du 30 août 2008.

23. Cuttitta P., "Le monde-frontière. Le contrôle de l'immigration dans l'espace globalisé ", Cultures \& Conflits, nº8, 2007, pp. 61-84. 
non militaire, et représentent donc une question marginale face aux menaces de terrorisme.

C'est pourquoi la première tâche des soldats est d'identifier les personnes interceptées pour déterminer s'il s'agit de migrants, et plus particulièrement de demandeurs d'asile, ou d'individus présentant des risques pour la sécurité du pays. Aussi plusieurs Soudanais ont-ils évoqué devant nous les recommandations des passeurs :

«Traversez la frontière en marchant vers les lumières ; quand l'armée israélienne passe, agenouillez-vous tout de suite et dites que vous demandez l'asile politique et les soldats vous emmèneront en lieu sûr et ne vous reconduirons pas en Egypte. »

Wolde se souvient pourtant :

«Quand nous sommes arrivés de l'autre côté, un des soldats parlait l'arabe et j'ai eu peur que nous soyons encore en Egypte; mais c'était un soldat israélien! »

Louis pensait quant à lui être perdu, ayant marché bien longtemps dans l'obscurité lorsque des soldats israéliens sont arrivés en voiture avec des fusils braqués sur lui; il a immédiatement mis les mains en l'air et a crié «Ivoirien! ».

Les soldats et gardes-frontières ont ordre de ne pas s'occuper des migrants - en dehors du fait de leur apporter les premiers soins - et de les remettre au plus vite aux services pénitentiaires israéliens. Dans les faits, cela peut prendre un, voire deux jours, et c'est donc aux soldats qu'incombe le premier accueil des migrants forcés. D’après tous les témoignages recueillis, c'est l'hospitalité des soldats qui étonnent le plus nos interlocuteurs. Beaucoup racontent qu'ils les ont menés à leur base militaire, les ont nourris, leur ont donné un lit, leur ont laissés se doucher et, surtout, ont soigné leurs blessures et ont même évacué par hélicoptère les blessés graves vers l'hôpital israélien le plus proche... D’ailleurs, les soldats en poste à la frontière israélo-égyptienne, dont certains réservistes sont médecins ${ }^{24}$, ont réclamé davantage d'équipement médical pour soigner le nombre grandissant de blessés recueillis après leur traversée. Ce n'est qu'après ces premiers jours que les migrants sont en principe conduits dans les centres de détention où il sera décidé de leur sort.

24. Tous les Israéliens sont contraints d'effectuer chaque année jusqu'à l'âge de 45 ans un mois par an de service comme « réserviste » dans l'unité dans laquelle ils ont effectué leur service militaire obligatoire de 3 ans, d'où le fait que les réservistes exercent différentes professions dans la vie civile, comme celle de médecin. 
C'est ainsi que, tous les mois, plusieurs centaines d'individus parviennent à traverser la frontière, prêts à risquer la mort pour échapper à une situation en Egypte, ou ailleurs, que beaucoup décrivent comme insupportable. Pour ces migrants forcés, «passer » en Israël signifie un franchissement vers un monde libre, démocratique et prospère qui leur attribuera des droits et mettra fin à leurs errances. Mais Israël représente également un Etat juif dont on ignore presque tout, pays-tabou pour certains (tels les Soudanais) ou terre promise chrétienne pour d'autres. Beaucoup y voient avant tout un lieu de protection où l'on obtient aisément des documents empêchant l'arrestation ou l'expulsion et où «les réfugiés sont bien traités ». Louis, qui n'avait jamais envisagé de migrer en Israël, avait entendu au Caire que tous les Ivoiriens en Israël recevaient immédiatement une protection temporaire... Il n'avait pas imaginé un instant entrer clandestinement dans un pays et traverser à pied une frontière de barbelés, nous expliquait-il en nous montrant ses blessures aux jambes. Les rumeurs circulent également au Caire concernant une vie meilleure en Israël, où l'on peut facilement trouver du travail et gagner de l'argent, un attrait certain pour soutenir les familles restées au pays ou ailleurs. Elias, qui a vécu deux ans en Egypte après sa fuite d'Erythrée, se souvient qu'on racontait que les salaires étaient beaucoup plus élevés en Israël, ce qui l'a décidé à entreprendre ce voyage au cours duquel l'un des ses amis a trouvé la mort en franchissant la frontière. Quant aux Egyptiens eux-mêmes, beaucoup sont persuadés qu'Israël recherche ces migrants pour exploiter économiquement cette main d'œuvre bon marché ${ }^{25}$. La frontière israéloégyptienne devient ainsi une frontière politique, économique, juridique et culturelle entre deux mondes.

Si, vu d'Egypte, «Israël, c'est le paradis» comme nous l'a expliqué un jeune Somalien, ces représentations de l'espace par delà la frontière sont aussi véhiculées par ceux qui sont réfugiés en Israël. Ainsi, un jeune rappeur du Sud-Soudan, arrivé depuis plus d'un an en Israël, a intitulé une de ses dernières chansons «IDF » (Israel Defense Forces) en hommage aux soldats israéliens qui l'ont recueilli à la frontière. Il affirme : "Quand nous avons traversé la frontière, c'est un jour que je n'oublierai jamais! ». Par ailleurs, le thème de la «sortie » biblique d'Egypte est souvent évoqué par nos interlocuteurs ${ }^{26}$.

Pourtant ce rêve d'un monde meilleur peut se briser et, pour beaucoup, la réalité de la situation des demandeurs d'asile en Israël, les laisse souvent amèrement déçus dès leur arrivée, à commencer par leur incarcération, arbitraire dans bien des cas. 


\section{Dispositifs de détention et espaces de circulation}

Si c'est le plus souvent l'armée qui intercepte les migrants forcés et qui les conduit au centre de détention le plus proche, les plus chanceux sont relâchés, faute de place dans les prisons, et d'autres encore parviennent à gagner les premières villes israéliennes, au Sud du Néguev, sans jamais rencontrer de soldats israéliens. C'est ainsi que ces zones frontalières deviennent des espaces de circulation grandissante de migrants forcés qui marchent au bord des routes ou attendent des bus. Une politique de détention pour toute personne ayant traversé irrégulièrement la frontière est certes en vigueur mais, en raison de l'afflux de migrants forcés depuis 2004 déjà, les prisons israéliennes sont débordées et l'armée reçoit souvent l'ordre de laisser les « infiltrateurs » à une station de bus en plein désert.

En revanche, le principe de non-refoulement est respecté et le « retour à chaud » (akhzara hama en hébreu) vers l'Egypte, à savoir l'expulsion immédiate à la frontière sans possibilité de demander l'asile, n'a eu lieu qu'une seule fois : en août 2007, 48 demandeurs d'asile subsahariens ont été reconduits à la frontière et, malgré les assurances de l'Egypte promettant de garantir leur sécurité, ils ont été détenus au secret plusieurs mois, interrogés et, pour la plupart renvoyés dans leur pays d'origine. Depuis cet épisode, et bien que le président Hosni Mubarak affirme accepter en retour tout individu qui traverse la frontière israélienne, cette pratique répressive, durement critiquée par des ONG israéliennes, ne s'était pas reproduite. Cette coopération transfrontalière se borne d'ailleurs à des accords informels de réadmission et il n'existe ni patrouilles conjointes ni échanges d'information. Cependant, entre le 23 et le 29 août 2008, un nouveau « renvoi immédiat » par les soldats israéliens a refoulé vers l'Egypte 91 demandeurs d'asile du Soudan, d'Erythrée et de Somalie, sans possibilité de recours quant à la décision d'expulsion ${ }^{27}$. Cette pratique, contraire au droit international ${ }^{28}$, démontre ici la « flexibilisation introvertie » de la frontière dans la mesure où elle acquiert la capacité de s'étirer dans l'espace jusqu'au lieu où l'étranger est arrêté 29 . De plus, depuis juin 2008, l'Egypte a expulsé plus de 1000 demandeurs d'asile érythréens vers leur pays, à l'encontre des recommandations du HCR, remettant davantage en question son statut de "pays sûr ». C'est pourquoi nombre d'associations de défense des droits de l'Homme ont manifesté en septembre dernier à Tel-Aviv contre ces refoulements, avec pour

27. Grinberg M. et al., «A l'encontre de son engagement envers la Cour Suprême : Tsahal expulse à nouveau les infiltrateurs vers l'Egypte ", Ha'aretz, 29 août 2008 (en hébreu).

28. Le refoulement de demandeurs d'asile et de réfugiés contredit l'engagement pris par Israël, signataire de la Convention de 1951 sur le statut des réfugiés (notamment article 33) et de la Convention des Nations unies contre la torture et autres peines ou traitements cruels, inhumains ou dégradants, de ne pas renvoyer d'individus dans des pays où ils risqueraient leur liberté, leur vie ou encourraient la torture, qu'il s'agisse du pays vers lequel ils sont refoulés ou tout autre pays vers lequel ils seraient renvoyés par la suite.

29. Cuttitta P., op. cit. 
slogan «Barak [Ministre de la Défense] envoie les réfugiés vers leur mort!». Pourtant, si les demandeurs d'asile africains sont souvent représentés par les militants israéliens comme des victimes, ayant souffert à la fois de persécutions dans leur pays d'origine et de brutalités en Egypte, notamment à la frontière, l'accueil qu'Israël leur réserve, à commencer par leur détention, témoigne du peu de reconnaissance de ce statut de victime.

\section{Ktziot : un centre de détention improvisé}

Pour faire passer un message clair - Israël ne veut pas de migrants forcés non juifs sur son territoire - l'Etat a mis en place de nouvelles formes de répression, telles le refoulement, bien qu'exceptionnel, et plus régulièrement la détention dans des prisons du pays. Si l'infraction à la législation concernant l'entrée irrégulière sur le territoire est punie de détention, la majorité des migrants forcés sont détenus en vertu de la Loi pour prévenir l'infiltration, une loi d'urgence datant de 1954 destinée aux «ressortissants d'un pays ennemi » qui peuvent, pour raisons de sécurité, être incarcérés de manière illimitée et sans instruction juridique ${ }^{30}$.

Outre cette criminalisation des demandeurs d'asile, dont certains fuient des persécutions et des génocides, la détention équivaut à amalgamer la recherche d'un refuge à un crime et devient de plus en plus contestée par la société civile israélienne et les ONG locales de défense des droits de l'Homme ${ }^{31}$, bien que l'Etat trouve une justification légale à leur rétention, à l'instar d'autres pays comme Malte ${ }^{32}$. Les détenus eux-mêmes expriment souvent leurs protestations par des grèves de la faim, voire des contestations plus violentes.

Mais, très vite, les services pénitentiaires ne peuvent plus « accueillir » l'afflux sans précédent de migrants forcés qui franchissent la frontière israéloégyptienne et le gouvernement décide, en juillet 2007, de créer dans l'urgence un centre de détention, attenant à la prison pour «détenus sécuritaires » de Ktziot, où sont incarcérés 2500 Palestiniens. Ce lieu d'enfermement sur le site

30. Une pétition a été présentée en mars 2006 à la Cour suprême d'Israël par l'ONG israélienne Hotline for Migrant Workers et la Refugee Clinic de la Faculté de droit de l'université de TelAviv en invoquant l'illégitimité de la discrimination opérée selon la nationalité. Un mois après, la Cour suprême a ordonné à l'Etat de trouver des solutions de détention alternatives - telle l'assignation à résidence dans des collectivités agricoles. Mais, aujourd'hui, les conditions de libération sont très facilement réunies.

31. En référence à l'article 31 de la Convention relative au statut des réfugiés, le Comité exécutif du HCR rappelle que la détention de réfugiés et demandeurs d'asile devrait être évitée, sauf dans quatre cas : pour vérifier l'identité ; pour déterminer le fondement d'une demande d'asile ; lorsqu'un individu a détruit ou présenté de faux documents ; et pour protéger la sécurité nationale et l'ordre public (UNHCR ExCom Conclusion n44, Detention of Refugees and AsylumSeekers, UNHCR, 37e session, 1986).

32 . Rodier C., Teule C., «Enfermement des étrangers : l'Europe sous la menace du syndrome maltais », Cultures $\mathcal{E}$ Conflits, n`57, 2005, pp. 119-155. 
de Ktziot - la prison la plus proche de la frontière avec l'Egypte - est présenté comme un «camp de réfugiés » temporaire pour distinguer les demandeurs d'asile des migrants économiques devant être expulsés. Ce «camping» en plein désert du Néguev, comme la presse l'a ironiquement surnommé ${ }^{33}$, détenait, au pic de son activité (été 2007), 1200 migrants forcés, y compris des femmes et des enfants (d'origine soudanaise et érythréenne) pour plusieurs mois. Ce centre de rétention improvisé, clôturé par des barbelés, se compose de tentes et de maisons préfabriquées, proches des bâtiments de la prison, où ont été installés des bureaux de l'administration pénitentiaire (y compris pour les juges d'instruction et les travailleurs sociaux), une infirmerie, une aire de jeux, une tente avec une télévision, des douches et des toilettes et, plus récemment, une salle de classe pour les enfants. Si seuls le HCR et l'ONG israélienne d'assistance juridique Hotline for Migrant Workers sont autorisés à visiter ce site, Ktziot fait l'objet d'une importante visibilité, que ce soit au travers des médias ou des mobilisations associatives qui y dénoncent les nombreuses violations des droits de l'Homme ${ }^{34}$. Ces violations amènent ainsi l'Etat israélien à détenir, pour la première fois de son histoire, des enfants et même des nouveau-nés puisque plusieurs femmes ont été transportées pour accoucher à l'hôpital de Beershéva puis ramenées après 3 jours au « camp » de Ktziot. Bien que des relations amicales se tissent entre gardiens et migrants au point que de jeunes mères ont parfois donné à leurs nourrissons les prénoms de leurs geôliers israéliens - il s'agit bien d'un régime disciplinaire avec un comptage trois fois par jour et la liberté de circuler uniquement dans l'enceinte assignée. En effet, un espace réservé aux hommes et un autre aux femmes et aux enfants sépare les familles de l'intérieur - car ils ne peuvent se rencontrer - et de l'extérieur - dans le cas où seul un conjoint ou un parent est détenu. Aujourd'hui, on compte environ 1000 migrants forcés détenus à Ktziot et quelques centaines dans les prisons à travers le pays.

Ce camp improvisé de Ktziot, ainsi que la création d' ' ailes pour étrangers » dans les prisons israéliennes pour criminels de droit commun n'est pas sans rappeler les nombreux camps de détention pour demandeurs d'asile et migrants irréguliers dans des pays comme la Grande-Bretagne, les Pays-Bas, la France ou l'Australie. Au départ, ces espaces d'enfermement d'étrangers sont pensés comme des solutions d'urgence provisoires mais prennent peu à peu un caractère durable avec leur pérennisation ${ }^{35}$, tout comme il existe en Israël un projet de construction de bâtiment en «dur » à Ktziot. Pourtant, si

33. Wurgraft N., "Comme une colo sauf que c'est à Ktziot », Ha'aretz, 17 août 2007 (en hébreu).

34. En janvier 2008, l'ONG Hotline for Migrant Workers a demandé en justice la fermeture de ce site, ce qui a été refusé sous prétexte que l'ONG ne proposait pas de solution alternative. Voir, sur les conditions de détention dénoncées comme inhumaines à Malte, Rodier C., Teule C., op. cit.

35. Fischer N., «Entre urgence et contrôle. Eléments d'analyse du dispositif contemporain de rétention administrative pour les étrangers en instance d'éloignement du territoire », TERRA Editions, coll. «Esquisses » (http://terra.rezo.net/article560.html). 
ces dispositifs de répression utilisés par Israël ont un but de dissuasion, la détention systématique est loin d'être suivie en pratique et le rapatriement n'est, pour le moment, pas à l'ordre du jour, pour la majorité des migrants forcés. Ainsi, plus qu'un instrument de contrôle migratoire, le centre de détention a d'abord un rôle symbolique en rappelant aux migrants qu'ils sont des étrangers « indésirables ", pour reprendre la formule de Michel Agier, et en démontrant le pouvoir de l'Etat relatif au droit au séjour sur son territoire. En effet, les logiques de libération sont souvent arbitraires et libérer des places pour détenir à leur tour ceux qui viennent tout juste de franchir la frontière transforme ces centres de détention en espaces de semi-circulation. Comme le remarque Chowra Makaremi 36 concernant les «zones d'attente » à l'aéroport de Roissy, il s'agit d'espaces intermédiaires de la circulation qui sont des espaces d'enfermement paradoxaux, créés par les contraintes de la circulation. En ce qui concerne Ktziot, depuis juillet 2007, 5000 migrants forcés de 25 pays africains y ont été détenus ${ }^{37}$.

Si pour Paolo Cuttitta, le centre de détention est une manifestation punctiforme de la frontière physique en territorialisant la frontière du statut, le pouvoir territorial investit quant à lui les frontières symboliques représentées par les statuts, en accentuant les statuts existants et en en créant de nouveaux dans les espaces urbains et ruraux de la circulation.

\section{Nouveaux statuts et nouvelles frontières dans les espaces de circulation}

Pour les migrants forcés qui échappent à la détention, soit parce qu'au moment de leur arrestation près de la frontière il n'y a plus de place dans les prisons, soit parce qu'ils parviennent à atteindre Beershéva, Eilat ou Tel-Aviv sans se faire arrêter, l'arrivée n'est pas toujours des plus chaleureuses. Ils sont en effet consternés devant l'absence d'infrastructures gouvernementales d'accueil et se voient souvent contraints de dormir dans des parcs publics et de survivre de dons alimentaires d'ONG ou d'initiatives privées, tout en se cachant jusqu'à l'obtention de documents de protection du HCR, sous peine d'être arrêtés. Certains sont hébergés quelques jours par des familles bédouines du Néguev, mais la plupart regagnent au plus vite Tel-Aviv, où ont été récemment mis en place par une ONG d'anciens demandeurs d'asile et réfugiés africains (ARDC) des abris d'urgence. Quelques églises, la plupart fondées par des migrants africains, accueillent aussi ponctuellement ceux qui arrivent de la frontière 38 .

36. Makaremi C., «Pénalisation de la circulation et reconfigurations de la frontière : le maintien des étrangers en “zones d'attente" », Cultures $\mathcal{E}$ Conflits, n71, automne 2008, pp. 55-73.

37 . Reznick R., " African Refugees Comprise 28\% of Tuberculosis Sufferers in Israel », Ha'aretz, 4 septembre 2008.

38. De façon générale, on assiste depuis 2007 à une mobilisation spontanée en faveur des « réfugiés africains » dans la société civile (voir Anteby-Yemini, L., op. cit.) 
Regagner Tel-Aviv est une priorité afin de déposer une demande d'asile au bureau du HCR. En effet, la communication entre les migrants forcés en Egypte et en Israël est constante, que ce soit par téléphone ou par messagerie électronique. Ainsi, les proches ou les amis déjà réfugiés en Israël expliquent aux candidats à la traversée comment passer, quand venir, quoi dire, et surtout comment se rendre, en premier lieu, à Tel-Aviv. Samuel, originaire de Somalie, raconte à ce titre qu'en arrivant à la frontière, les soldats israéliens l'ont laissé partir pour Beershéva et, de là, des étudiants lui ont donné de l'argent pour prendre le bus en direction de Tel-Aviv, comme on lui avait recommandé au Caire. On nous a même cité des migrants qui arrivent directement de la frontière égyptienne au HCR à Tel-Aviv dans la même journée. Certains sont munis de leur portable égyptien et communiquent donc rapidement avec leurs proches en Israël ; ils maintiennent également des liens avec ceux restés en Egypte, que ce soit par le téléphone, l'Internet ou les transferts monétaires (dans les deux sens). Ces moyens de communication, courants dans toutes les migrations transnationales, jouent un rôle majeur pour alimenter ou réduire le flux migratoire, car si le sort d'un groupe particulier n'est pas propice, tel des Guinéens qui n'ont pu obtenir de protection et dont beaucoup ont été détenus plusieurs mois en Israël, l'information circule et la filière peut cesser rapidement.

Nul besoin de dire que, par conséquent, le HCR est débordé, avec en moyenne 30 demandes par jour et des effectifs qui ont été doublés en moins de quatre ans. Il n'y a toujours pas de législation concernant l'asile en Israël : c'est le HCR qui traite les demandes et propose à un comité interministériel du gouvernement israélien les dossiers sélectionnés. Ce comité les valide le plus souvent, octroyant dans la plupart des cas (Erythréens, Soudanais, Congolais) une protection temporaire à titre humanitaire avec un visa de séjour, et parfois de travail, pour six mois à un an renouvelable ${ }^{39}$. Cependant, le taux de reconnaissance du statut de réfugiés tel qu'établit par la Convention de Genève de 1951 est l'un des plus bas au monde (0,5 \%), en partie parce que le gouvernement n'autorise pas l'accès à cette procédure aux ressortissants d'un pays ennemi. Dans un premier temps toutefois, la majorité des demandeurs d'asile obtient des documents de protection du HCR durant l'examen de leur demande et peuvent enfin circuler pour rechercher un emploi et un logement, qu'ils partagent bien souvent avec d'autres compatriotes. Aussi les quartiers sud de Tel-Aviv deviennent-ils des lieux de concentration privilégiés, en raison des bas loyers, des opportunités d'emplois, de la proximité d'ONG et de services (commerces ethniques, centres de téléphonie, églises africaines). D'autres « réfugiés » travaillent dans les hôtels de la station balnéaire d'Eilat ou dans des collectivités agricoles (moshavim ou kibbutzim) au

39. Sur la procédure d'asile en Israël, voir Akoka K., « La demande d'asile en Israël : vers la “nationalisation" de la procédure de prise en charge », communication au colloque international «L’asile au Sud : Afrique, Méditerranée... », Ouagadougou, Burkina-Faso, juin 2006. 
Nord et au Sud du pays. Ainsi se constituent peu à peu des communautés de migrants forcés africains mais avec de nouvelles frontières, entre statuts juridiques cette fois.

En effet, le chaos juridique, politique et administratif, ou ce que Sarah Willen nomme la «turbulence gouvernementale 40 », dans laquelle l'Etat gère ces migrants forcés, avec la détention d'une minorité (environ 1500 ) et la libre circulation des autres (plus de 12500 ) génère des situations et des statuts bien souvent incohérents et arbitraires. Ainsi, Wolde, d'Erythrée, a été arrêté à la frontière et détenu près de Tel-Aviv plusieurs semaines avant d'être libéré par le HCR, alors que Samuel, d'origine somalienne, avait lui été hébergé par les soldats israéliens avant qu'ils ne l'abandonnent à une station de bus. Quant à Sophie, une Ivoirienne interceptée par l'armée, elle est depuis quatre mois en détention à Ktziot ${ }^{41}$. Nelly, une Soudanaise est arrivée à Tel-Aviv sans jamais être arrêtée. Enfin, Ismail, un Guinéen débouté par le HCR, est dans une prison proche de Tel-Aviv depuis deux ans. Aussi, trouve-t-on aujourd'hui des demandeurs d'asile détenus plusieurs mois, plusieurs années ${ }^{42}$ ou simplement quelques jours, alors que d'autres ne passent par aucun centre de détention; certains reçoivent des documents de protection et même des visas de travail alors que d'autres n'en reçoivent pas; d'autres encore ont reçu une protection temporaire mais elle a cessé ou cessera sous peu et sont donc en danger d'arrestation ou d'expulsion, comme le sont les 100000 migrants économiques irréguliers estimés en Israël, dont la plupart sont entrés par l'aéroport de TelAviv avec un visa de touriste de 3 mois ou un permis de travail pour un temps limité, qu'ils ont dépassé. Or, Israël mène depuis 2002 une politique répressive de lutte contre l'immigration irrégulière non juive : entre 2002 et 2005, 140000 migrants de travail ont été reconduits aux frontières, soit par un rapatriement « volontaire » soit par des expulsions ${ }^{43}$.

Dans un tel contexte, il se crée donc des frontières symboliques entre statuts (demandeurs d'asile en attente, déboutés, réfugiés, détenteurs d'une protection temporaire, titulaires d'un permis de résidence, sans statut) mais aussi entre nationalités et leurs privilèges. Ainsi, seuls les ressortissants de la

40. Willen S., «L'hyperpolitique du "Plus jamais ça !" : demandeurs d'asile soudanais, turbulence gouvernementale et politiques de contrôle des réfugiés en Israël ", Cultures $\mathcal{E}$ Conflits, n ${ }^{\circ} 71$, automne 2008, pp. 93-112.

41. Comme ailleurs, un migrant qui fait une demande d'asile selon les termes de la convention de Genève peut rester détenu jusqu'à l'issue de cette procédure, parfois très longue. Sur Malte, voir Rodier C., Teule C., op. cit.

42 . Il s'agit surtout de demandeurs d'asile déboutés qui refusent de rentrer dans leur pays, qui n'ont pas de documents de voyage permettant aux autorités israéliennes de les expulser et dont les pays d'origine n'entretiennent pas de liens diplomatiques avec Israël ou dont l'ambassade en Israël du pays dont ils se réclament ne les reconnait pas ; ils restent parfois ainsi sans possibilité d'être libérés ni expulsés.

43. Willen S. (ed.), Transnational Migration to Israel in Global Comparative Context, Lexington Books, Lanham, 2007. 
République démocratique du Congo reçoivent actuellement prima facie une protection temporaire en raison des conflits dans leur pays ; quant aux demandeurs d'asile érythréens, on leur octroie depuis janvier 2008 des titres de séjour et des permis de travail renouvelables. Les Soudanais, eux, obtiennent des visas de séjour mais sans autorisation de travail, bien qu'un emploi soit toléré ; par ailleurs, une mesure gouvernementale exceptionnelle a attribué à 600 demandeurs d'asile du Darfour des permis de résidence en raison d'une «affinité de souffrance $44 »$. En revanche, les Ivoiriens ne recevront plus de protection temporaire à compter de décembre 2008, ce qui compliquera la libération de ceux détenus. Enfin, les ressortissants des autres pays africains sont souvent déboutés et demeurent sans statut, comme nombre de Maliens ou Guinéens.

Cependant, si certains droits comme la scolarisation n'opèrent pas de distinction entre les enfants de statut différent, ni les demandeurs d'asile ni ceux munis d'une protection temporaire ne reçoivent d'assistance financière ou d'assurance médicale; cette absence de droits sociaux les rend vulnérables et accentue davantage leur position aux marges de la société israélienne. Par ailleurs, les frontières territoriales restent elles aussi fermées, aussi bien aux titulaires d'une protection temporaire puisqu'ils ne peuvent voyager sans risquer de ne plus être ré-admis en Israël, qu'aux déboutés dont certains ne peuvent être expulsés. Ils restent donc incontestablement dans une situation de liminalité (border-state) que ce soit face aux frontières symboliques des droits ou aux frontières territoriales du pays. Nous sommes donc en droit de nous demander si Isräl représente une étape migratoire ou une destination finale pour ces migrants forcés. Or, si l'Etat israélien leur octroie des protections temporaires pour éviter la sédentarisation, beaucoup souhaitent simplement y trouver un refuge avant de rêver aux chemins vers l'Europe...

Pour conclure, il n'existe toujours pas de politique israélienne claire envers les migrants forcés arrivant par l'Egypte, qu'il s'agisse de directives de détention ou de mesures d'accueil dans la ville. Ces pratiques contradictoires proviennent d'une double logique : sécuritaire d'une part, avec le projet de construction d'un mur le long de la frontière, et humanitaire d'autre part, avec l'octroi de permis de résidence et de protections temporaires à certains. Ces incohérences découlent sans doute de la nouveauté et de l'ampleur du phénomène qui place dorénavant Israël dans le jeu du contrôle et de la gestion de flux migratoires non juifs en provenance d'un pays tiers. Il est donc confronté pour la première fois au phénomène des migrations terrestres clandestines par une frontière qui n'avait jamais été pensée comme frontière migratoire. Trois points s'ensuivent : 
- Mutation dans la fonction de la frontière : de la sécurité intérieure à la migration internationale

Pour Israël, sa frontière avec l'Egypte passe d'une frontière à l'origine sécuritaire, entre pays ennemis jusqu'aux accords de paix de 1979 et du retrait israélien du Sinaï, à une frontière de migration irrégulière en provenance de pays subsahariens transitant par ce pays frontalier. De même que les significations attribuées aux frontières nationales changent avec le temps, le contexte politique, le statut et l'identité des individus qui les franchissent ${ }^{45}$, ici ce sont les migrants forcés, l'opinion publique et l'Etat qui redéfinissent des images concurrentes de cette frontière et de ceux qui la traversent, que ce soit en tant que frontière-protection pour des réfugiés persécutés qu'il faut accueillir ou en tant que frontière-tampon contre une invasion de migrants de travail. Ainsi, avec l'évolution du trafic et le changement dans la nature même des passages frontaliers, la frontière israélo-égyptienne représente un nouvel enjeu géopolitique pour les deux pays : contenir les migrations forcées africaines.

\section{- Nouvel enjeu de la frontière et redéfinition du rôle de l'Etat : surveillance} et pouvoir

En effet, l'Egypte et Israël surveillent et contrôlent de façon accrue leur zone frontalière, les Egyptiens pour empêcher les migrants de quitter le territoire égyptien, les Israéliens pour les intercepter à leur entrée en territoire israélien. Rappelons, comme le soulignent Donnan et Wilson, que les zones frontalières sont des sites et des symboles de pouvoir, de souveraineté et de sécurité, et qu'une anthropologie des frontières est aussi une analyse anthropologique des relations de pouvoir entre deux Etats-nations ${ }^{46}$. Dans notre cas, les deux Etats exercent leur pouvoir dans ces espaces par le recours à la détention, l'expulsion ou l'usage excessif de la force. Toutefois, ces nouvelles préoccupations, civiles et non plus militaires, ne sont pas sans créer des tensions à la frontière ni des tensions diplomatiques entre les deux pays. Ainsi, l'Egypte doit adopter une politique répressive à la demande d'Israël et, en ce sens, devient, comme d'autres pays de transit, "l'instrument de la flexibilisation extravertie de la frontière du pays de destination 47 ». On pourrait presque parler de délocalisation de l'asile lorsque l'Egypte se voit contraint de contenir les migrants forcés en deçà de la frontière israélienne et de prendre ses responsabilités vis-à-vis de ses frontières avec le Soudan, entre autres. Le Sinaï peut en l'occurrence être comparé au Sahara, transformé par : 
"l'embrigadement forcé des pays maghrébins dans le rôle de "sentinelles avancées" sommées de jouer le rôle de barrage de rétention et de sous-traitant d'une répression “déportée” et "délocalisée” loin des frontières et des opinions européennes 48 ».

Pour le moment, il n'existe pas d'accords formels entre Israël et l'Union européenne pour qu'Israël admette sur son territoire les demandeurs d'asile ou mette en place des camps de rétention comme au Maghreb ou en Libye ${ }^{49}$.

\section{- Globalisation de l'asile et déplacement des frontières $d u$ "Nord»}

A l'heure actuelle, il ne s'agit pas d'Egyptiens qui traversent la frontière (à l'exception de quelques centaines), mais plutôt de ressortissants d'autres pays $\mathrm{du}$ «Sud » qui voient la frontière avec Israël comme une porte d'entrée vers une terre de refuge politique et d'opportunités économiques de par sa situation géopolitique. On pourrait, dans une certaine mesure, opérer un parallèle avec la frontière américano-mexicaine pour les migrants du Guatemala, de la Bolivie ou du reste de l'Amérique latine qui tentent de la franchir. On pourrait même rejoindre Peter Andreas en disant que le Sinaï devient «les frontières externes de la nouvelle Europe 50 ». Et s'il est question d'une nouvelle «frontière externalisée » de l'Europe au Moyen-Orient, certains politiciens parlent même de la frontière Soudan/Egypte qui se déplace vers le Nord... A ce titre, dans le cas où, comme le laisse entendre le ministre israélien de l'Intérieur, un mur serait construit pour "endiguer le flot de migrants irréguliers 51 », les routes terrestres pourraient bien devenir maritimes à l'avenir, ajoutant à la diversification constante des itinéraires et à la globalisation de la migration forcée qui placent désormais Israël sur la carte de ces circulations transnationales.

L'avenir du passage clandestin par la frontière israélo-égyptienne dépend en partie de la politique de répression ou d'accueil qu'adoptera en fin de compte le gouvernement israélien. En mars 2008, le Premier ministre Ehud Olmert avait déclaré vouloir arrêter les «infiltrateurs de travail » en employant "raisonnablement la force si nécessaire ", tout en déplorant l'insuffisance des mesures de contrôle égyptiennes ${ }^{52}$. En mai 2008 passait en première lecture au Parlement israélien une proposition d'amendement de la loi

48. Bensaad A., op. cit., p. 24.

49. En revanche, le ministre israélien de la Justice observe que son pays ne peut accueillir tous les « réfugiés africains » et suggère de négocier avec l'Europe pour définir combien de réfugiés pourraient être reçus par les Européens et combien par les Israéliens. Voir Mizrahi S., «Le ministre Friedman : il faut se comporter avec indulgence envers les réfugiés du Darfour », Maariv, 30 mai 2007 (en hébreu).

50. Andreas P., op. cit.

51. I. Shahar, “Bar-On Calls African Refugees Seeping in from South Major Risk”, Ha'aretz, 19 juin 2007.

52. Jerusalem Post, 24 mars 2008. Voir Frenkel S., op. cit. 
sur la Prévention de l'infiltration sanctionnant tout individu traversant clandestinement la frontière à des peines allant de 5 à 7 ans de détention. Mais, simultanément, Israël octroie des permis de séjour et de travail à de nombreux demandeurs d'asile, se justifiant en tant qu'Etat juif d'une obligation morale et d'un devoir historique d'assistance humanitaire à ceux qui fuient des persécutions. C'est ainsi qu'on assiste à l'instrumentalisation de la frontière par certaines ONG comme en témoigne un clip mis en ligne par l'ACRI (Association pour les droits civils en Israël) ${ }^{53}$ qui titre, sur fond de musique menaçante :

«Les réfugiés ont fui pour sauver leurs vies [...]. Les survivants ont réussi à atteindre l'autre côté. Les soldats les ont renvoyés à ceux qui leur tiraient dessus. Ce n'est pas de l'histoire. C'est l'armée israélienne (IDF) qui le fait en ce moment à des réfugiés qui traversent la frontière sud d'Israël. L'asile pour les réfugiés. C'est un droit [...] c'est notre devoir. »

On voit ainsi comment la fonction même de la frontière peut être radicalement transformée et de quelle manière cette frontière confronte Israël non seulement à un défi migratoire mais également à un défi éthique. 